\title{
Venetic Personal Anthroponyms
}

\author{
Rainhardt S. Stein ${ }^{1}$, Giancarlo T. Tomezzoli² \\ ${ }^{1}$ Universiteit Utrecht, Utrecht, Holland \\ ${ }^{2}$ Etno-Archaeological Observatory, Munich, Germany \\ Email: rstein@uu.nl,gt21949@gmx.de
}

How to cite this paper: Stein, R. S., \& Tomezzoli, G. T. (2020). Venetic Personal Anthroponyms. Archaeological Discovery, 8, 135-146.

https://doi.org/10.4236/ad.2020.82008

Received: February 10, 2020

Accepted: February 29, 2020

Published: March 3, 2020

Copyright $\odot 2020$ by author(s) and Scientific Research Publishing Inc. This work is licensed under the Creative Commons Attribution International License (CC BY 4.0).

http://creativecommons.org/licenses/by/4.0/

\begin{abstract}
Our investigation has been conducted on a set of 854 Venetic personal anthroponyms from the area corresponding to the today's Veneto/Friuli and areas around, derived from the Corpus Inscriptionum Latinarum a continuously updated Latin inscription catalogue covering the Roman Empire period and its provinces. The Venetic anthroponyms appear deeply Latinised and the root of each Venetic personal anthroponym was identified by comparing it with corresponding lexemes in the present surviving Slavic languages. It results that the Venetic personal anthroponyms having sure Slavic roots in the set is $23.89 \%$ of the derived Venetic personal anthroponyms, which permits to estimate that during the Roman Empire period, i.e. about five cen., roughly about $24 \%$ of the Venetic population had Slavic ascendancies. This highlights that Slavs were already present in the today's Veneto/Friuli and in said areas around well before the Roman Empire period and the VII century A.D. of the generally accepted theory of the Slavs late arrival in Europe. The logical consequence is that this theory is wrong and should be rejected.
\end{abstract}

\section{Keywords}

Veneti, Anthroponyms, Slavs, Late Arrival, Latinization, Roman Empire, Corpus Inscriptionum Latinarum, Linguistics

\section{Introduction}

Ancient Veneti tribes coming from Caucasus populated different regions of the today's Europe: Armorica (FR), Constance Lake (DE), Lazio (IT), Veneto (IT), Slovenia (SI), Austria (AU), Vistula river delta (PL) and Paphlagonia (TU). A Veneti tribe, named also Eneti, was considered to be part of the Illyrian ethnic community (Herodotus, 2013: I.196) and inhabited the region around the North Adriatic Sea.

Later, it was explained that "The part of the plain near the Adriatic had never 
ceased to be in the possession of another very ancient tribe called the Veneti, differing slightly from the Gauls in customs and costume and speaking another language. About these people the tragic poets tell many marvellous stories." (Polybius, 2016: II.17.5-6). In our opinion, the expression "very ancient tribe" is a clear indication that this Veneti tribe was considered an autochthonous population of the northern Italy. The expression "speaking another language" is an indication that Veneti were a people distinguished from the Celts.

On the basis of information acquired by Meandrius, Veneti, named Eneti, allied to the Trojans, migrated from Paphlagonia to the North Adriatic Sea, after the Troy fall (Strabo, 1903: XII.3.25). Meandrius's information contains certainly something of true, but most probably Veneti/Eneti came to North Italy not by chance, but seeking refuge by their relatives. In fact, it is possible that the autochthonous Veneti (Polybius, 2016: II.17.5-6) inhabited the North Adriatic region before the Trojan War.

Venetic anthroponyms can be encountered in some publications (Pellergini\& Prosdocimi, 1967; Šavli, Bor, \& Tomažič, 1996) which do not deal with them in a systematic way. Exception is a publication (Pauli, 1891) in which they are listed systematically according to their appearance in the Corpus Inscriptionum Latinarum $(C I L)$. However, this last publication does not consider their etymology.

\section{Venetic Personal Anthroponyms}

The above mentioned publication (Pauli, 1891), on pages 352 - 378, identifies 854 sure Venetic (V.) personal anthroponyms in either nominative, or genitive, or dative case and highlights them with a bold initial capital character. Pages 352 - 359 contain V. anthroponyms from the area corresponding to the today's Veneto/Friuli. Pages 359 - 378 contain V. anthroponyms from areas around the today's Veneto/Friuli: Istria, Liburnia, Dalmatia, Pannonia superior, Pannonia inferior and Noricum.

In our analysis, said V. anthroponyms were considered one by one and only those having sure Slavic roots were retained in alphabetic order in Table 1 together with four other CIL. anthroponyms, indicated by * in Table 1, not recognized as V. in said publication but having sure Slavic roots.

Each record in Table 1 comprises the progressive record number (Re.), the V. anthroponyms (V. Anth.) sharing the same Slavic root, the corresponding root meaning (R. Mean.), the corresponding lexemes in present surviving Slavic languages (Sl. Lexem.) from which the root meaning was inferred, the respective $C I L$. (CIL) entry and the page (Page) in said publication where each V. anthroponym resides. Records with $C I L$. entries more than the numbers of the $\mathrm{V}$. anthroponyms indicate that at least one $\mathrm{V}$. anthroponym is cited in more than one CIL. entry. Records with CIL. entries less than the number of the corresponding $\mathrm{V}$. anthroponyms indicate that at least one CIL. entry cites more than one V. anthroponym. Table 1 should be read bearing in mind the language conventions of Table 2. 
Table 1. Venetic anthroponym list.

\begin{tabular}{|c|c|c|c|}
\hline Re. V. Anth. & R. Mean. & Sl. Lexem. & Page \\
\hline \multicolumn{4}{|c|}{ A } \\
\hline 1 & \multicolumn{3}{|c|}{$\begin{array}{l}\text { Adens: hell-Bos. Had, Blg. ад, Cro. Had, O.Ch.Sl. адъ, Rus. ад, Ser. хад, Ukr. ад; } \\
\text { CIL. III, 1. no. 4332, p. } 300 .\end{array}$} \\
\hline 2 & \multicolumn{3}{|c|}{$\begin{array}{l}\text { Arovinto: ardent-Bos. žarki, Blg. жарък, Cro. žarki, Pol. żarliwy, Rus. жаркий, } \\
\text { Ser. жарки, Slv. horlivý, Ukr. жаркий; CIL. III, 1. no. 2993, p. } 364 .\end{array}$} \\
\hline 3 & \multicolumn{3}{|c|}{$\begin{array}{l}\text { Atressa, Atresso, Atressus: quick-Bos. hitar, Cro. hitar, Lit. atrs, O.Blg. пАдръ, Rus. } \\
\text { хитрый, Ser. хитар, Slo. hitro; CIL. III, 1. no. 3380, p. 369; CIL. III, 1. no. 3687, p. } \\
\text { 370; CIL. III, 2. no. 5275, p, } 374 .\end{array}$} \\
\hline 4 & \multicolumn{3}{|c|}{$\begin{array}{l}\text { Ausco: ears-Bel. вушкі, Bos. uši, Blg. ушите, Cro. uši, Cze. uši, Lit. ausis, Mac. } \\
\text { уши, Pol. uszy, Rus. уши, Ser. уши, Slo. ušesa, Slv. uši; CIL. III, 2. no. 5265, p. } 374 .\end{array}$} \\
\hline 5 & $\begin{array}{l}\text { Avetonia, Avet } \\
\text { јава; CIL. III, } 2 \\
\text { p. } 375 ; \text { CIL. III, }\end{array}$ & $\begin{array}{l}\text { Ivii/u/, Avvae: reality-Bos. java, } \\
0 \text {, p. } 377 \text {; CIL. III, 2. no. 5571, p. } \\
\text { 563, p. } 377 \text {. }\end{array}$ & $\begin{array}{l}\text { us. явь, Ser. } \\
\text { I, 2. no. } 5422 \text { a, }\end{array}$ \\
\hline
\end{tabular}

Basio, Baesio, Baezo, Bauso, Bizo: without-Bel. без, Bos. bez, Blg. без, Blg.dial. бизая, Cro. bez, Cze. bez, Lit. be, Mac. без, Pol. bez, Rus. без, Ser. без, Slo. brez, Slv. bez, Ukr. 6e3; CIL. V, 1. no. 2887, p. 355; CIL. III, 1. no. 3437, p. 370; CIL. III, 1. no. 2781, p. 362; CIL. III, 2. no. 4889, p. 371; CIL. III, 1. no. 2782, p. 362.

Bateiae, Bateli, Bato, Bataronis, Batonis, Batti, Boto: brother-Bel. брат, Bos. brate, Blg. брат, Cro. brat, Cze. bratr, Lit. brolis, Mac. брат, Pol. brat, Rus. брат, Ser. брате, Slo. brat, Slv. brat, Ukr. брат; CIL. III, 2. no. 5031, p. 373; CIL. III, 2. no. 5057, p. 373; CIL. III, 1. no. 4276, p. 368; CIL. III, 1. no. 3558, p. 370; CIL. III, 1. no. 2749, p. 365; CIL. III, 1. no. 3286, p. 369; CIL. III, 1. no. 3558, p. 370; CIL. III, 1. no. 3593, p. 370; CIL. III, 2. no. 5191, p. 374.

Bellia: white-Bel. бел, Bos. bela, Blg. бял, Cro. bijela, Cze. bílý, Lit. balta, Mac. бело, Pol. biały, Rus. белый, Ser. беo, Slo. bela, Slv. biely, Ukr.білі; CIL. III, 2. no. 5155 , p. 374 .

Beria: trust/faith—Bel. давер, Bos. poverenje, Blg. вяра, Cze. důvěra, Mac. доверба, Rus. вера, Ser. поверење, Slv. dôvera, Ukr. довіру; CIL. V, 1. no. 947, p. 357.

Besna, Bisena: spring-Bel. вясна, Lit. versmé, Rus.весна, Ukr. весна; CIL. III, 1. no. 4597 , p. 369 ; $C I L$. V, 1. no. 1363 , p. 358.

Boi, Boiscus: fighter-Bel. баец, Bos. borac, Blg. боец, Cro. borac, Cze. bojovník, Mac. борец, Pol. bojowiec, Rus. боец, Ser. војник, Slo. borec, Slv. vojak, Ukr.6оєць; CIL. III, 1. no. 3816, p. 367; CIL. V, 2. no. 8112, 17, p. 355.

Boto, Bottia, Bottiae, Bottionis, Botucae, Butonis, Butto, Buttonis: water-Bel. вада, Bos. voda, Blg. вода, Cro. voda, Cze. voda, Мac. вода, O.Ch.Sl. вода, Pol. woda, Rus. вода, Ser. вода, Slo. voda, Slv. voda, Ukr. вода; CIL. III, 2. no. 5191, p. 374; CIL. III, 2. no. 4864, p. 371; CIL. III, 2. no. 4915, p. 371; CIL. III, 2. no. 6495, p. 373; CIL. III, 1. no. 3826, p. 367; CIL. III, 1. no. 3819, p. 367. берег, Slv. breh, Ukr. берег; CIL. V, 1. no. 3729, p. 354.

Bucca, Bucci, Buccio, Bucioni, Buco, Buctor, Buctoris: beech-Bel. бук, Bos. bukva, Blg. бук, Cro. bukva, Cze. buk, Lit. buko, Мас. бука, Pol.buk, Rus. бук, Ser. буква, Slo. bukev, Slv. buk, Ukr. бук; CIL. III, 1. no. 3788, p. 366; CIL. III, 1. no. 3787, p. 366; CIL. III, 1. no. 3789, p. 366; CIL. III, 1. no. 3823, p. 367. 


\section{Continued}

Boudes: awake-Bel. абуджаны, Bos. budan, Blg. буден, Cro. budan, Cze. vzbudit se, Lit. pabudęs, Мас. буден, Pol. budzić, Rus. будить, Ser. будан, Slo. buden, Slv. bdiet', Ukr. будити; CIL. III, 2. no. 5477, p. 376.

Buio, Buioni, Buiio, Buioni, Buionis: rowdy-Bel. буян, Rus. буян; CIL. III, 1. no. 3790, p. 366; CIL. III, 1. no. 3855, p. 367; CIL. III, 1. no. 3826, p. 367; CIL. III, 1. no. 3860, p. 367; CIL. III, 1. no. 3826, p. 367; CIL. III, 1. no. 3866, p. 367.

Burrani, Burrus: storm-Blg. буря, Cze. bouřka, Mac. бура, Pol. burza, Rus. буря, Slv. búrka, Ukr. буря; CIL. III, 2. no. 5463, p. 376, CIL. III, 2. no. 5418, p. 375.

\section{$\mathrm{C}$}

Caliai, Calsasia, Calupa, Colla: mud-Bos. kal, Blg. кал, Cro. kal, Mac. кал, Ser. кал, Slo. kal, Ukr. кал; CIL. V, 1. no. 3840, p. 354; CIL. V, 1. no. 2414, p. 354; CIL. III, 2. no. 5061, p. 373; CIL. V, 1. no. 491, p. 361.

Coma, Comargus: stone-Bel. камень, Bos. kamena, Blg. камък, Cro. kamen, Cze. kámen, Lit. akmuo, Мас. камен, Pol. kamień, Rus. камень, Ser. камен, Slo. kamen, Slv. kameň, Ukr. камінь; CIL. III, 2. no. 4899a, p. 371; CIL. III, 1. no. 3158, p. 366.

Carmo: root-Bel. корань, Bos. korijen, Blg. корен, Cro. korijen, Cze. kořen, Lit. kastis, Мас. корен, Pol. korzeń, Rus. корень, Ser. корен, Slo. koren, Slv. koreň, Ukr. корінь; CIL. III, 2. no. 5644, p. 377.

Cicca: honor-Bos. čast, Blg. чест, Cro. čast, Cze. čest, Mac. чест, Pol. cześć, Rus. честь, Ser. част, Slo. čast, Slv. čest', Ukr. честь; $C I L$. V, 1. no. 1422, p. 359.

Cona: horse-Bel. конь, Bos. konj, Blg. кон, Cro. konj, Cze. kůn̆, Мас. коњ, Pol. koń, Rus. конь, Ser. коњ, Slo. konj, Slv. kôň, Ukr. кінь; CIL. III, 2. no. 5311, p. 374.

Craili: the end-Bos. kraj, Blg. край, Cro. kraj, Mac. крај, Rus. край, Ser. крај; CIL. III, 1. no. 4376, p. 368.

Cupiti, Cupitus*: to buy-Bel. купляць, Bos. kupiti, Blg. купувам, Cro. kupiti, Cze. koupit, Мac. купи, Pol. kupić, Rus. купить, Ser. купити, Slo. kupiti, Slv. kúpit, Ukr. купувати; CIL. III, 2. no. 4889, p. 371; CIL. III, 2. no. 5151, p. 374.

\section{$\mathrm{D}$}

Dacisco: to oppress-Bos. tlačiti, Cro. tlačiti, Rus. давить, Ser. тлачити, Slo. tlačiti, Ukr. давити; CIL. V, 1. no. 1047, p. 358.

Dasi, Dassio: to give-Bel. даваць, Bos. dati, Blg. дадеш, Cro. dati, Cze. dát, Lit. duoti, Mac. дава, Pol. dawać, Rus. дать, Ser. дати, Slo. dajati, Slv. dat', Ukr. давати; CIL. III, 1. no. 3162 b, p. 366; CIL. III, 1. no. 2305, p. 365.

Derva, Dervoniae: tree-Bel., Bos. drvo, Blg. дърво, Cro. drvo, Mac. дрво, Pol. drzewo, Rus. дерево, Ser. дрво, Slo. drevo, Ukr. дерево; CIL. III, 2. no. 5419, p. 375; CIL. III, 1. no. 3659, p. 370.

Deusa, Deusi, Deusonis: soul—Bel. душа, Bos. duša, Blg. душа, Cro. duša, Cze. duše, Lit. dvasia, Mac. душа, Pol. dusza, Rus. душа, Ser. душа, Slo. duša, Slv. duše, Ukr. душа; CIL. III. 2. no. 5425, p. 375; CIL. III, 2. no. 5370, p. 375; CIL. III, 2. no. 5426, p. 375.

Dasi, Diso, Disocno, Disocni, Dizo: the tenth-Bel. дзясяты, Bos. deseti, Blg. десетата, Cro. deseti, Cze. д а хум, Lit. dešimtas, Mac. десеттиот, Pol. dziesiąty, Rus. десятый, Ser. десети, Slo. deseto, Slv. desiaty, Ukr. десятий; CIL. III, 1. no. 3162b, p. 366; CIL. III, 2. no. 5322, p. 374; CIL. III, 2. no. 5076, p. 373; CIL. V, 1 . no. 893 , p. 357 . 


\section{Continued}

Dumba: oak—Bel. дуб, Blg. дуб, Cze. dub, Mac. даб, Pol. dąb, Rus. дуб, Slv. dub, Ukr. дуб; CIL. III, 2. no. 5289, p. 374.

Ettro: nucleus-Bel. ядро, Bos. jezgra, Blg. ядро, Cro.jezgra, Cze. jádro, Mac. јадро, Pol.jądro, Rus. ядро, Ser.jезгро, Slo. jedro, Slv. jadro, Ukr.ядро; CIL. V, 1. no. 1133 , p. 358

Gannico: to hunt-Bos. goniti, Blg. гоня, Cro.goniti, Cze. hon, Lit. guiti, O.Ch.Sl. гонити, Pol. uganiać, Rus. гнать, Ser. гонити, Slv. hon, Ukr. гнати; CIL. III, 2. no. 5102, p. 373.

Getaciae, Getacio, Getacius: harvest-Bel., Bos. žetva, Blg. жътва, Cro. žetva, Mac. жетва, Rus. жатва, Ser. жетва, Slo. žetev, Slv. žatva, Ukr. жати; CIL. V, 1. no. 603, p. 362; CIL. V, 1. no. 554, p. 362.

Glago: verb-Bos. glagol, Blg. глагол, Cro. glagol, Мac. глагол, O.Ch.Sl. глаголъ, Rus. глагол, Ser. глагол, Slo. glagol; CIL. V, 1. no. 3679, p. 353.

Gnavo: anger-Bel. гнеў, Bos. gnjev, Blg. гняв, Cro. gnjev, Cze. hněv, Мас. гнев, Pol. gniew, Rus. гнев, Ser. гнев, Slv. hnev, Ukr. гнів; CIL. III, 2. no. 4725, p. 370.

\section{$\mathbf{H}$}

Hostilia: to remain/to stay-Bos. ostati, Blg. остават, Cro. ostati, Cze. zůstat, Mac. остане, Rus. остаться, Ser. остати, Slo. ostati, Slv. ostat; CIL. V, 1. no. 2066, p. 389.

Iariovidius: ardent—Bos. žarki, Blg. жарък, Cro. žarki, Pol. żarliwy, Rus. жаркий, Ser. жарки, Slv. horlivý, Ukr. жаркий; CIL. V, 1. no. 3908, p. 352.

Iavi, Iavia, Iavolenus: to appear-Bel. 3'явіцца, Bos. pojaviti, Blg. явявам ce, Cro. pojaviti, Cze. objevit, Мас.појавува ce, Pol. pojawić się, Rus. появляться, Ser. појавити, Slo.pojaviti, Slv. javí, Ukr. з'явитися; CIL. III, 1. no.2781, p. 362; CIL. V, 1. no. 3487 , p. 353.

Iettus, Intona, Ioiti, Itionis, Itto, Ittu, Ituli, Itul(i), Ittunis : to go-Bel. icцi, Bos. ići, Blg. отида, Cro. ići Cze. jít, Lit. eiti, Mac. Да оди, O.Ch.Sl. ити, Pol. iść, Rus. идти, Ser. иде, Slo. iti, Slv. íst', Ukr. йти; CIL. III, 1. no. 2768, p. 362; CIL. III, 2. no. 5133, p. 374; CIL. III, 2. no. 5131, p. 374; CIL. III, 2. no. 5242, p. 374; CIL. III, 2. no. 5640, p. 377; CIL. III, 2. no. 5505, p. 376; CIL. III, 2. no. 4934, p. 372; CIL. III, 2. no. 5425, p. 375; CIL. III, 2. no. 5489, p. 376; CIL. III, 2. no. 5640, p. 377.

\section{$\mathrm{J}$ \\ K}

Kanius: horse-Bel. конь, Bos. konj, Blg. кон, Cro. konj, Cze. kůn̆, Mас. коњ, Pol. koń, Rus. конь, Ser. коњ, Slo. konj, Slv. kôň, Ukr. кінь; CIL. V, 1. no. 992, p. 358; CIL. V, 2. no. 8307, p. 358.

\section{$\mathbf{L}$}

Laepici, Laepicus, Laepius, Laepocae, Laepocus: nice-Bos. lijepo, Cro. lijep, Rus. лъпока, Ser. леп, Slo. lepo, Ukr лепський; CIL. III, 1. no. 2922, p. 363; CIL. III, 1. no. 2431, p. 365; CIL. III, 1. no. 3804, p. 366; CIL. V, 1. no. 453, p. 361; CIL. III, 1. no. 3322 , p. 369 . 
Laevica, Laevicus, Lavi: lion—Bel. леў, Bos. lav, Blg. лъв, Cro. lav, Cze. lev, Lit. liūtas, Mac. лав, Pol. lew, Rus. лев, Ser. лав, Slo. lev, Slv. lev, Ukr. лев; CIL. V, 1. no. 449 , p. 360; $C I L$. V, 1, no. 449, p. 361; CIL. III, 1. no. 2792, p. 362.

Lannae, Lanno, Lannus: deer-Bel. алені, Bos. jelen, Blg. елен, Cro. jelen, Cze. jelen, Lit. elnias, Мас. елен, Pol. jeleń, Rus. олень, Ser. јелен, Slo. jelen, Slv. jeleň, Ukr. олень; CIL. V, 1. no. 3655, p. 353.

Lediae: ice-Bel. лёд, Bos. led, Blg. лед, Cro. led, Cze. led, Lit. ledas, Мас. лед, Pol. lód, Rus. лёд, Ser. лед, Slo. led, Slv. lad, Ukr.лід; CIL. III, 2. no. 4743, p. 371.

Licnos, Licnus: personal-Bos. lični, Blg. личен, Cro. lični, Мас. лично, Rus. личный, Ser. лични; CIL. V, 1. no. 21, p. 359; CIL. V, 1; no. 1818, p. 357.

Lucania, Lucco*, Luccoia, Lucano, Lucanus, Lucconia: bow-Bel. лук, Bos. luk, Blg. лък, Cro. luk, Lit. lankas, Mac. лак, Pol. łuk, Rus. лук, Ser. лук, Slo. lok, Slv. lúk, Ukr. лук; CIL. III, 1. no. 3599, p. 370; CIL. V, 1. no. 523; CIL. III, 2. no. 5019, p. 372; CIL. III, 1. no. 4599, p. 369; CIL. III, 1. no. 4599, p. 369.

\section{$\mathbf{M}$}

Malabanus, Malai, Malos, Malsonis, Maltini, Mollico, Molligemia: small-Bel. маленькі, Bos mali, Blg. малък, Cro. mali, Cze. malý, Mac. мал, Pol. mały, Rus. малый, Ser. мали, Slo. majhen, Slv. malý, Ukr. малий; CIL. V, 1. no. 150, 359; CIL. III, 2. no. 5419, p. 375; CIL. III, 2. no. 5698, p. 378; CIL. III, 1. no. 3214, 9, p. 363; CIL. V, 1. no. 587 , p. 362.

Mataronis, Meter, Metra: to throw-Blg. мятам, Rus. метать, Slo. metanje, Slv. metat', Ukr. метати; CIL. V, 1. no. 2608, p. 355; CIL. V, 1. no. 618, p. 362.

Mendae: lesser-Bel. менш, Bos. manja, Cro. manji, Cze. menší, Rus. меньший, Slo. manjši, Slv. menšie, Ukr. менший; CIL. III, 1. no. 3144, p. 364.

Mor(ano), Morano: sea-Bel. мopa, Bos. more, Blg. мope, Cro. more, Cze. moře, Mac. мope, Pol. morze, Rus. мope, Ser. мope, Slo. morje, Slv. more, Ukr. mope; CIL. V, 1. no. 8197, p. 362.

Mosgaito: brain-Bel. мозг, Bos. mozak, Blg. мозък, Cro. mozak, Cze. mozek, Мас. мозок, Pol. mózg, Rus. мозг, Ser. мозак, Slo. možgani, Slv. mozog, Ukr. мозок; CIL. III, 2. no. 5033, p. 373.

Mosicu, Musonis: man-Bos. muž, Blg. мъж, Cro. muž, Cze. muž, Pol. mąż, Rus. муж, Ser. муж, Slv. muž, Ukr. муж; CIL. III, 2. no. 5373, p. 375; CIL. III, 2. no. 5365, p. 375.

\section{$\mathbf{N}$}

\section{$\mathrm{O}$}

Obilo, Obilie: plentiful—Bos. obilan, Blg. обилен, Cro. obilan, Мас. обилен, Pol. obfity, Rus. Ukr. обильный, Ser. обилан, Slo. obilen, Ukr. обильний; CIL. III, 2. no. 5664, p. 377; CIL. III, 2. no.6503, p. 373.

Obuciori: beech-Bel. бук, Bos. bukva, Blg. бук, Cro. bukva, Cze. buk, Lit. buko, Мас. бука, Pol. buk, Rus. бук, Ser. буква, Slo. bukev, Slv. buk, Ukr. бук; CIL. III, 1. no. 3790 , p. 366 .

Occi: eyes-Bel. вочы, Bos. oči, Blg. очи, Cro. oči, Cze. oči, Lit. akys, Mac. очи, O.Ch.Sl. чи, Pol. oczy, Rus. очи, Ser. очи, Slo. oči, Slv. oči, Ukr. очi; CIL. III, 2. no. 4987, p. 372.

Ophus, Opiae, Opiaus: experienced-Blg. опитен, Cze. osvědčený, Pol. obyty, Rus. опытный; CIL. III, 1. no. 3322, p. 369; CIL. III, 1. no. 3144, p. 364; CIL. V, 1. no. 320 , p. 360 . 


\section{Continued}

Ostiala: to remain/to stay-Bos. ostati, Blg. остават, Cro. ostati, Cze. zůstat, Mac. остане, Rus. остаться, Ser. остати, Slo. ostati, Slv. ostat', Ukr. ; CIL. V, 1. no. 2906, p. 355 .

Ovi, Ovincii: sheep.-Bel. авечка, Bos. ovce, Blg. овца, Cro. ovca, Cze. ovce, Lit. avys, Мас. овца, Pol. owca, Rus. овца, Ser. овца, Slo. ovca, Slv. ovca, Ukr. овець; CIL. III, 1. no. 3796, p. 366; CIL. III, 2. no. 5139, p. 374.

Paeticus: the fifth-Bel. пяты, Bos. peti, Blg. петият, Cro. peti, Cze. pátý, Mac. петтиот, Pol. piąty, Rus. пятый, Ser. пети, Slo. peti, Slv. piaty, Ukr. п'ятий; CIL. V, 1. no. 2035 , p. 356 .

Poia, Poio: to drink-Bel. піць, Bos. piti, Blg. да пия, Cro. piti, Cze. pít, Mac. да пие, Pol. pić, Rus. пить, Ser. пити, Slo. piti, Slv. pit', Ukr. пити; CIL. III, 1. no. 2994, p. 364; CIL. III, 2. no. 5020, p. 372.

\section{Q}

$\mathbf{R}$

Rabutio: slave-Bel. paб, Bos. rob, Blg. poб, Cro. rob, Mac. poб, O.Ch.Sl. paбa, Rus. paб, Ser. poб, Ukr. paб; CIL. V, 1. no. 3679, p. 353.

Raedo: joy-Bel. радасць, Bos. radost, Blg. радост, Cro. radost, Cze. radost, Mac. радост, Pol. radość, Rus. радость, Ser. радост, Ukr. радість; CIL... V, 2. no. 8320, p. 359.

Rumae, Rumnis: red-Bos. rujan, Blg. румен, Cro. rujan, Cze. rudý, Lit. rudas, Pol. rumiany, Rus. румяный, Ser. рујан, Slo. rdeča, Slv. ryšavý, Ukr. рудий; CIL. III, 2. no. 5350, p. 375; CIL. III, 2. no. 4966 , p. 372.

Runcasiae: hand/arm-Bel. рука, Bos. ruka, Blg. ръка, Cro. ruka, Cze. ruka, Lit. ranka, Мас. рака, Pol. ręka, Rus. рука, Ser. рука, Slo. roka, Slv. ruka, Ukr. рука; CIL. V, 1. no. 3437, p. 353.

\section{$S$}

Sebacauso: dog-Bel. сабака, Cze. skoba, Lit. šuo, Rus. собака, Ukr. собака; CIL. III, 2. no. 5027, p. 372.

Samanna, Sami, Samianta, Samicantuni, Sammucin(us), Samuco, Samudae: alone-Bos. sam, Blg. сам, Cro. sam, Cze. sám, Mac. сам, Pol. sam, Rus. сам, Ser. сам, Slo. sam, Slv. sám, Ukr. caм; CIL. III, 1. no. 2610, p. 365; CIL. V, 1. no. 1208, p. 358; CIL. III, 2. no. 5550, p. 377; CIL. III, 2. no. 5480, p. 376; CIL. V, 1. no. 3253, p. 353; CIL. III, 2. no. 4971, p. 372; CIL. III, 2. no. 5365, p. 375.

Sato: hundred-Bel. сто, Bos. stotinu, Blg. сто, Cro. сто, Pol. sto, Rus. сто, Ser. сто, Slo. sto, Slv. sto, Ukr. сто; CIL. III, 2. no. 5465, p. 376.

Scali: rock-Bel. скала, Blg. скала, Cze. skála, Pol. skała, Rus. скала;Slo. skala, Slv. skala, Ukr. скеля; CIL. III, 1. no. 4582, p. 369.

Secco, Siccai: cleaver-Bel. секача, Blg. секира, Cze. sekáček, Lit. skustuvas, Mac. секач, Rus. секач, Slv. sekáčik; CIL. III, 1. no. 3810, p. 367; CIL. V, 1. no. 366, p. 360.

Silanus*:force-Bel. сіла, Bos. silu, Blg. сила, Cro. sila, Cze. síla, Мас. сила, Pol. siła, Rus. сила, Ser. сила, Slo. silo, Slv. sila, Ukr. сила; CIL. V, 1. no. 3437, p. 353. 


\section{Continued}

71

72

73

74

75

76
Soliae, Soleiae: salt-Bel. соль, Bos. soli, Blg. сол, Cro. sol, Cze. sůl, Mac. сол, Pol. sól, Rus. соль, Ser. co, Slo. sol, Slv. sol, Ukr. сіль; CIL. III, 2. no. 5487, p. 376; CIL. V, 1. no. 1264, p. 358.

Sporilla: dispute-Bel. спрэчка, Bos. spor, Blg. спор, Cze. spor, Mac. спор, Pol. spór, Rus. спор, Ser. спор, Slo. spor, Slv. spor, Ukr. спірка; CIL. III, 2. no. 5441, p. 376.

Suri, Surinus, Suro, Suronis, Surus, Surato: severe-Bel. суровы, Blg. суров, Pol. surowy, Rus. суровый, Slv. surový, Ukr. суворий; CIL. III, 2. no. 5084, p. 373; CIL. III, 2. no. 5418, p. 375; CIL. V, 1. no. 483, p. 361; CIL. III, 1. no. 3821, p. 367; CIL. III, 2. no. 5095, p. 373; CIL. III, 2. no. 6513, p. 372; CIL. III, 1. no. 2753, p. 365; $C I L$. III, 2. no. 5153, p. 374.

Tamacus: darkness-Bel. цемра, Bos. tama, Blg. тъмнина, Cro. tama, Cze. tma, Lit. tamsa, Mac. темнина, Rus. тьма, Ser. тама, Slo. tema, Slv. tma, Ukr. темрява; CIL. III, 2. no. 5080, p. 373.

Tottiae, Totto, Totulo: the one-Cze. ten, Lit. tą, Rus. тот, Slv. ten; CIL. III, 2. no. 5383, p. 375; CIL. III, 2. no. 5479, p. 376; CIL. III, 2. no. 5485, p. 376.

Treioni, Tritaneri, Tritano, Tritanoni, Tritanonis, Triti, Tritus: the third-Bel. трэці, Bos. treći, Blg. третият, Cro. treći, Cze. třetí, Lit. trečioji, Мac. третиот, Pol. trzeci, Rus. третий, Ser. трећи, Slo. tretji, Slv. tretia, Ukr. третій; CIL. III, 2. no. 6401, p. 365; CIL. III, 1. no. 2796, p. 363; CIL. III, 1. no. 2792, p. 362; CIL. III, 2. no. 6351, p. 365; CIL. III, 1. no. 2793, p. 363; CIL. III, 2. no. 6412, p. 363; CIL. III, 1. no 3058, p. 364; CIL. III, 2. no. 6411, p. 363.

Tergitio: bargain-Bel. торг, Cze. targ, Pol. targ, Rus. торг; CIL. III, 1. no. 4231, p. 368.

\section{$\mathrm{U}$}

Uttu: to go-Blg. отивам, Cze. jít, Lit. eiti, Мac. оди, Rus. идти, Ser. иди, Slo. iti, Ukr. йти; CIL. III, 2. no. 5523, p. 376.

\section{$\mathrm{V}$}

Vanni: bath-Bel. банята, Blg. баня, Lit. vonia, Mac. бања, Rus. баня, Ukr. баня; CIL. III, 2. no. 5421, p. 375.

Varbilo: willow-Bel. вярба, Bos. vrba, Blg. върба, Cro. vrba, Cze. vrba, Mac. врба, Pol. wierzba, Rus. верба, Ser. врба, Slo. vrba, Slv. vŕba, Ukr. верба; CIL. III, 1. no. 4600 , p. 369.

Velleco: great/giant-Bel. вялікі, Bos. velik, Blg. велик, Cro. velik, Cze. skvělý, Pol. wielki, Rus. великий, Ser. велики, Slo. velik, Slv. skvelý, Ukr. великий; CIL. III, 2. no. 5425 , p. 375.

Vendae, Vendo, Vendoni: to lead-Bos. voditi, Blg. води, Cro. voditi, Cze. vedení, Lit. vadovauti, Мас. да води, Rus. водити, Ser. водити, Slo. voditi, Slv. vodit', Ukr. водити; $C I L$. V, 1. no. 3425, p. 353; CIL. III, 1. no. 2796, p. 363; CIL. III, 1. no. 2797; p. 363; CIL. III, 2. no. 6352, p. 365.

Vesclevesis: glory-Bel. слава, Bos. slava, Blg. слава, Cro. slava, Cze. sláva, Lit. šlove, Mac. слава, Pol. sława, Rus. слава, Ser. слава, Slo. slava, Slv. sláva, Ukr. слава; CIL. III, 1. no. 3058, p. 364. 


\section{Continued}

84

Vinioca, Vinioco, Vinonnio: wine-Bel. віно, Bos. vino, Blg. вино, Cro. vino, Cze. víno, Lit. vyno, Mac. вино, Pol. wino, Rus. вино, Ser. вино, Slo. vino, Slv. víno, Ukr. вино; CIL. III. 1. no. 3154, p. 364; CIL. III, 1. no. 2502, p. 365.

Volteregi, Voltisa, Voltisae, Voltani: power-Bos. vlast, Blg. власт, Cro. vlast, Pol. władza, Rus. власть, Ser. власт, Ukr. влада; CIL. III, 1. no. 3823, p. 367; CIL. III. no. 2900, p. 363; CIL. III, 1. no. 2891, p. 363; CIL. III, 1. no. 3821, p. 367.

\section{Y W}

Z

Zaiio: rabbit-Bos. zec, Blg. заек, Cro. zec, Lit. zuikis, Mac. зајак, Pol., Rus. заяц, Ser. зец, Slo. zajec, Slv. zajac, Ukr заєць; CIL. III, 1. no. 2756, p. 365.

Table 2. Language conventions.

\begin{tabular}{cccc}
\hline Bel. Belarus & Bos. Bosnian & Blg. Bulgarian & Cro. Croatian \\
\hline Cze. Czech & Lit. Lithuanian & Mac. Macedonian & O. Blg. Old Bulgarian \\
O.Ch.Sl. Old Church Slavonic & Pol. Polish & Rus. Russian & Slo. Slovenian \\
Slv. Slovakian & Ser. Serbian & Ukr. Ukrainian & \\
\hline
\end{tabular}

\section{Discussion}

The CIL. is a continuously updated Latin inscription catalogue covering the period of the Roman Empire and its provinces, therefore, it is not surprising that Table $1 \mathrm{~V}$. anthroponyms are deeply Latinized. The Slavic root for each V. anthroponym was identified by comparing it with corresponding lexemes in the present surviving Slavic languages (Table 2), this because ancient Slavic documents are rare (Ambrozic \& Tomezzoli, 2003; Ambrozic, 2005; Ambrozic et al., 2006; Pellergini \& Prosdocimi, 1967; Šavli et al., 1996; Serafimov, 2006; Serafimov, 2007a; Serafimov, 2007b; Serafimov \& Tomezzoli, 2009; Serafimov \& Tomezzoli, 2012; Tomažič \& Tomezzoli, 2003; Tomezzoli, 2001; Tomezzoli \& Čudinov, 2002; Tomezzoli, Serafimov, \& Vodopivec, 2009; Tomezzoli \& Serafimov, 2013; Vodopivec, 2008; Vodopivec, 2009a; Vodopivec, 2009b) and normally contain few lexemes, insufficient for meaningful comparisons with Table $1 \mathrm{~V}$. anthroponyms.

\subsection{Initial Considerations}

The concept of nature is present in: T1.17 storm, T1.18 mud, T1.19 stone, T1.20 root, T1.22 horse, T1.23 the end, T1.27 tree, T1.30 oak, T1.31 nucleus, T1.40 horse, T1.42 lion, T1.43 deer T1.44 ice, T1.50 sea, T1.51 beech, T1.58 sheep, T1.63 red, T1.65 dog, T1.68 rock, T1.71 salt, T1.74 darkness, T1.80 willow T1.86 rabbit.

The concept of person is present in: T1.3 quick, T1.4 ears, T1.6 without, T1.9 trust/faith, T1.21 honor, T1.25 to oppress, T1.28 soul, T1.34 verb, T1.36 to remain/to stay, T1.39 to go, T1.41 nice, T1.45 personal, T1.47 small, T1.51 brain, T1.52 man, T1.55 eyes, T1.57 to remain/to stay, T1.60 to drink, T1.62 joy, T1.64 hand/arm, T1.70 force, T1.72 dispute, T1.78 to go, T1.79 bath, T1.81 great/giant, 
T1.83 glory, T1.86 power.

The concept of personality is present in: T1.2 ardent, T1.15 awake, T1.16 rowdy, $\mathrm{T} 1.35$ anger, $\mathrm{T} 1.37$ ardent, $\mathrm{T} 1.38$ to appear, $\mathrm{T} 1.53$ plentiful, T1.56 experienced, T1.73 severe, $\mathrm{T} 1.75$ the one.

The concept of family is present in: T1.7 brother, T1.29 the tenth, T1.49 lesser, T1.59 the fifth, T1.76 the third.

The concept of profession is present in: T1.11fighter, T1.24 to buy, T1.26 to give, T1.32 to hunt, T1.33 harvest, T1.46 bow, T1.48 to throw, T.61 slave, T1.67 hundred, T1.68 cleaver, T1.77 bargain, T1.82 to lead, T1.84 wine.

\subsection{Intermediate Considerations}

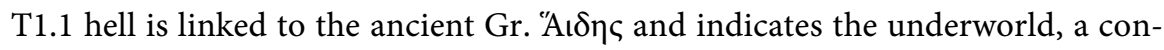
cept passed lately to the Christian tradition as hell.

T1.2 ardent, T1.37 ardent are linked to the theonym Iarovid/Iarovit indicating the Slavic fertility god, named also Jarilo. This theonym contains the Slavic roots jar: ardent, rod: give birth-Bos. roditi, Blg. раждам, Cro. roditi, Cze. porodit, Mac. роди, Pol. rodzić, Rus. рожать, Ser. породити, Slo. roditi, Slv. porodit, Ukr. Родити and vid/vit: to be-Bel. быць, Bos. biti, Blg. бъда, Cro. biti, Cze. být, Lit. būti, Мас. биди, Pol. być, Rus. быть, Ser. бити, Slo. biti, Slv. byt', Ukr. бути.

T1.3 quick is linked to the hydronyms Adriatic the sea facing the today's Veneto/Friuli, Adrias/Atrianus a no longer existing, ancient channel of the today Po river delta, mentioned by Hecateus, Theopompus and Ptolemy (Wikipedia, 2018) and Jantra a today's Blg. river, tributary of Danube, which was named Athrys by the ancient Thracians.

The hydronym Plavis, the ancient name of the today's Po River coasting the today's Veneto, is linked to the Slavic root to swim/to flow-Bel. плаваць, Bos. plivati, Blg. плувам, Cro. plivati, Cze. plavat, Lit. plaukti, Mac. плива, O.Blg. плавати, Pol. pływać, Rus. плавать, Ser. пливати, Slo. plavati, Slv. plávat', Ukr. плавати.

T1.4 ears, T1.14 beech, T1.25 to oppress, T1.32 to hunt, T1.46 bow, T1.47 small, T1.66 alone, T1.69 cleaver, T1.81 great/giant, T1.84 wine preserve the suffix-ko present in today's Slavic personal anthroponyms: Vinko, Stanko and Slavko. T1.10 spring, T1.22 horse, T1.66 alone preserve the suffix-na present in today's Slavic personal anthroponyms: Dragana, Stana and Svetlana.

\subsection{Final Considerations}

In said publication (Pauli, 1891), the V. anthroponyms in the area corresponding to the today's Veneto/Friuli are 211 and in said areas around are 643 for a V. anthroponym total of 854 which represents a good sample for a statistical consideration. The V. anthroponyms in Table 1 having sure Slavic roots are 204 which represent $23.89 \%$ of the $\mathrm{V}$. anthroponyms in said publication. This permits to estimate that during the Roman Empire period, i.e. about five cen., roughly $24 \%$ of the Venetic population had Slavic ascendancies. 


\section{Conclusion}

Said 24\% of Venetic population having Slavic ascendancies, in the today's Veneto/Friuli and said areas around during the Roman Empire period, highlights that Slavs were already present in the today's Veneto/Friuli and in said areas around well before the Roman Empire period and the VII century A.D. of the generally accepted theory of the Slavs late arrival in Europe. The logical consequence is that this theory is wrong and should be rejected.

\section{Acknowledgements}

We are grateful to Ms. Vostretsova G. for her kind revision of the present article.

\section{Conflicts of Interest}

The authors declare no conflicts of interest regarding the publication of this paper.

\section{References}

Ambrozic, A. (2005). The "Warrior" Stele from Lemnos. In Origin of the Slovenes. Proceedings of the Third International Topical Conference Ancient Settler of Europe (pp. 107-120). Ljubljana: Založništvo Jutro.

Ambrozic, A., \& Tomezzoli, G. (2003). The “Tavola da Este” Inscription. In Origin of the Slovenes. Proceedings of the International Workshop Traces of European Past (pp. 132-146). Ljubljana: Založništvo Jutro.

Ambrozic, A., Serafimov, P., \& Tomezzoli, G. (2006). The Venetic Inscription ES 120 on the Cup of "Scolo di Lozzo". In Origin of the Slovenes. Proceedings of the Fourth International Topical Conference Ancient Inhabitants of Europe (pp. 166-173). Ljubljana: Založništvo Jutro.

Herodotus (2013). The Histories I.196. Medford, MA: Tufts University. http://www.perseus.tufts.edu/hopper/text?doc=Perseus\%3Atext\%3A1999.01.0126\%3A book\%3D1\%3Achapter\%3D196

Pauli, C. (1891). Die Veneter und Ihre Schrftdenkmäler, Altitalische Forschungen. Leipzig: Johan Ambrosius Barth.

Pellergini, G. B., \& Prosdocimi, A. L. (1967). La lingua Venetica (Vol. 2). Padova: Istituto Glottologia Università di Padova.

Polybius (2016). Histories. II.17.5-6 (Classical Library ed., Vol. 1). Cambridge, MA: Harvard University Press 1922 thru 1927.

http://penelope.uchicago.edu/Thayer/E/Roman/Texts/Polybius/2*.html

Šavli, J., Bor, M., \& Tomažič, I. (1996). Veneti. First Builders of European Community. Wien: Editiones Veneti.

Serafimov, P. (2006). The Sitovo Inscription. In Origin of the Slovenes. Proceedings of the Fourth International Topical Conference Ancient Inhabitants of Europe (pp. 198-204). Ljubljana: Založništvo Jutro.

Serafimov, P. (2007a). Steinberg Inscription. In Origin of the Slovenes. Proceedings of the Fourth International Topical Conference Ancient Inhabitants of Europe (pp. 174-182). Ljubljana: Založništvo Jutro.

Serafimov, P. (2007b). Translation of the Eteocretan EPIOI Inscription. In Origin of the 
Slovenes. Proceedings of the Fifth International Topical Conference Origin of Europeans (pp. 176-183). Ljubljana: Založništvo Jutro.

Serafimov, P., \& Tomezzoli, G. (2009). The Inscription from Tell El-Dab'a. In Origin of the Slovenes. Proceedings of the Seventh International Topical Conference Origin of Europeans (pp. 89-96). Ljubljana: Založništvo Jutro.

Serafimov, P., \& Tomezzoli, G. (2012). New Reading of the Linear a Inscription on the Golden Pin CR-ZF-1 from Crete. In Origin of the Slovenes. Proceedings of the Tenth International Topical Conference Origin of Europeans (pp. 83-90). Ljubljana: Založništvo Jutro.

Strabo. Geography XII.3.25. Strabo, Geography H.C. Hamilton, Esq., W. Falconer, M.A., Ed. Somerville, MA: Tufts University. http://www.perseus.tufts.edu/hopper/text?doc=Perseus\%3Atext\%3A1999.01.0239\%3A book\%3D12\%3Achapter\%3D3\%3Asection\%3D25

Tomažič, I., \& Tomezzoli, G. (2003). The Inscription Pauli No. 39. In Origin of the Slovenes. Proceedings of the International workshop Traces of European Past (pp. 147-157). Ljubljana: Založništvo Jutro.

Tomezzoli, G. (2001). About Two Magre-Rhaetic Inscriptions in the Civic Natural History Museum in Verona. In Origin of the Slovenes. Proceedings of the First International Topical Conference the Veneti within the Ethnogenesys of the Central-European Population (pp. 182-187). Ljubljana: Založništvo Jutro.

Tomezzoli, G., \& Čudinov, V. A. (2002). The "Spada di Verona". In Origin of the Slovenes. Proceedings of the Conference Ancient Settlers of Central Europe (pp. 65-73). Ljubljana: Založništvo Jutro.

Tomezzoli, G., \& Serafimov, P. (2013). The Linear-A Inscriptions II.1, II.2 from the Palace of Knossos. In Origin of the Slovenes. Proceedings of the Eleventh International Topical Conference Origin of Europeans (pp. 105-114). Ljubljana: Založništvo Jutro.

Tomezzoli, G., Serafimov, P., \& Vodopivec, V. (2009). Two Noricum Inscriptions. In Origin of the Slovenes. Proceedings of the Seventh International Topical Conference Origin of Europeans (pp. 119-127). Ljubljana: Založništvo Jutro.

Vodopivec, V. (2008). Retijski Napis Pustertal. In Origin of the Slovenes. Proceedings of the Sixth International Topical Conference Origin of Europeans (pp. 137-141). Ljubljana: Založništvo Jutro.

Vodopivec, V. (2009a). Napisi na Ptujskih Olienkah. In Origin of the Slovenes. Proceedings of the Seventh International Topical Conference Origin of Europeans (pp. 141-147). Ljubljana: Založništvo Jutro.

Vodopivec, V. (2009b). Najdaljši Venetski Napis. In Origin of the Slovenes. Proceedings of the Seventh International Topical Conference Origin of Europeans (pp. 97-118). Ljubljana: Založništvo Jutro.

Wikipedia (2018). Adria (River). https://en.wikipedia.org/wiki/Adria_(river) 